\title{
Kernos
}

Revue internationale et pluridisciplinaire de religion grecque antique

9 | 1996

Varia

\section{La cité et ses composantes : remarques sur les sacrifices et la procession des Panathénées}

\section{Pierre Brulé}

\section{OpenEdition Journals}

\section{Édition électronique}

URL : http://journals.openedition.org/kernos/1156

DOI : 10.4000/kernos. 1156

ISSN : 2034-7871

\section{Éditeur}

Centre international d'étude de la religion grecque antique

\section{Édition imprimée}

Date de publication : 1 janvier 1996

ISSN : 0776-3824

\section{Référence électronique}

Pierre Brulé, «La cité et ses composantes : remarques sur les sacrifices et la procession des Panathénées », Kernos [En ligne], 9 | 1996, mis en ligne le 21 avril 2011, consulté le 21 avril 2019. URL: http://journals.openedition.org/kernos/1156; DOI : 10.4000/kernos.1156 
Kernos, 9 (1996), p. 37-63.

\section{La cité en ses composantes :}

\section{remarques sur les sacrifices et la procession \\ des Panathénées}

Que ne l'a-t-on répété : sur la frise du Parthénon, l'atelier de Phidias aurait sculpté non seulement la procession des Panathénées, mais le « dèmos luimême » ${ }^{1}$; nous retrouverions là « les différentes classes d'Athènes qui s'associent dans le culte commun rendu à leur patronne $»^{2}$... On n'en finirait pas de citer les opinions allant dans le même sens : les spectateurs du Ve siècle auraient retrouvé sur ce long bandeau sculpté l'image de leur corps social.

Pourtant ceux qui ont prêté une attention suffisante à la plus grande procession religieuse de la plus grande cité grecque, soit en considérant les quelques quatre cents personnages de la frise, soit (moins souvent) les sources littéraires conviendront aisément que l'image qui en résulte offre avec la conception que nous nous faisons du dèmos athénien bien des différences, voire des désaccords profonds, parfois de véritables oppositions. Citons quelques exemples: la place inhabituelle, incongrue, du féminin, celle de la jeunesse (et même de la vieillesse), et celle des non-citoyens, autant de traits qui sont sans écho dans la pratique sociale. Et il y aurait, dans le détail, d'autres divergences, tant et si bien qu'il ne serait pas inconcevable d'y voir même parfois un monde à l'envers.

Mais peut-être ne sont-ce là que formules où la rhétorique joue trop sa partie. Pour éclairer la question des rapports entre procession et société, il convient d'abord de réexaminer la documentation, en évitant, si possible, de se mettre dans la situation de chercher ce que l'on désire y trouver. Les difficultés herméneutiques sont déjà assez grandes pour qu'on n'aille pas y ajouter celles qui naissent de ce qu'on pourrait appeler une lecture préalable du sujet. Il en va ainsi, par exemple, de cet a priori fréquent consistant à n'appréhender l'objet - la frise - que dans sa seule dimension symbolique.

Il me semble, par ailleurs, qu'on ne prend pas assez garde à une difficulté majeure : le rôle joué par l'histoire. Malgré son caractère rituel, la fête est un

\footnotetext{
1 P. SCHMITT-PANTEL dans P. SCHMITT et Cl. ORIEUX, Histoire grecque, Paris, 1995, p. 237 : «La frise est ... une représentation du dèmos lui-même, dans une de ses activités religieuses : une procession. "
}

2 L. G. KAHIL, L'Acropole, in Athènes au temps de Périclès, Paris, 1964, p. 131. 
objet historique, elle change. Quittons la Grèce. On ne célèbre pas le 14 juillet de la même façon en 1790, à la fin du XIXe siècle et à la fin du XXe. Soit un programme s'articulant ainsi : retraite aux flambeaux - feu d'artifice - bal gratuit - réveil en fanfare - défilé et prise d'armes - jeux pour enfants - bal payant. Il est celui d'une petite ville de France des années cinquante du XXe siècle. Son enchaînement dit bien ce qu'une telle fête possède en commun avec d'autres manifestations festives, ainsi le défilé et la prise d'armes, mais aussi le bal payant; il dit ce qu'il a de spécifique : retraite aux flambeaux, réveil en fanfare, jeux de l'après-midi; il dit aussi ce qui est développement «tardif » : la pétarade qui, tout en perdurant, s'est amplifiée en feu d'artifice. Ces greffons ne prennent bien que s'ils se trouvent en harmonie avec l'esprit général de la fête. En harmonie avec l'ambiance. Car en tout temps et en tout lieu, la somme des rituels ne suffit pas à définir la fête.

Cela, la Grèce le vérifie. La trilogie procession - sacrifice - repas sacrificiel, qui constitue la structure la plus commune des cérémonies religieuses grecques, est susceptible de prendre, selon les lieux, les circonstances, la place dans la journée, les saisons, l'année, les groupes qui y prennent part, selon les divinités et les aitia, des couleurs extrêmement différentes, jusqu'à paraître opposées. Et ce qui est vrai s'agissant de l'ensemble, l'est aussi dans les détails : pour les éléments, les moments de la fête. La prise d'armes du 14 juillet a une tout autre saveur que celle du 11 novembre: alors que la seconde est uniquement militaire et sombre, participent à la première les pompiers, voire les sociétés de gymnastique, et elle est lumineuse. La commémoration de la prise de la Bastille et celle de la Fête de la Fédération commandent d'autres comportements que celle de l'Armistice de 1918. En termes grecs, on dirait que les aitia diffèrent.

Sans vouloir cultiver le paradoxe qui consisterait à prendre le contre-pied de l'opinion commune, il convient de se montrer plus circonspect en prêtant un peu plus d'attention à l'histoire afin de se poser la question des rapports entre la composition de la procession et celle de la société. On devrait, en cette matière, se souvenir des conseils que M.P. Nilsson dispensait à ceux qui voulaient rendre compte des rites grecs du mariage. Si, disait-il, chaque détail des synthèses que proposaient alors les érudits était juste - le rite existe réellement, à une certaine époque, en un certain lieu -, la fresque qui résultait de la juxtaposition de l'ensemble des détails tenait plus du kaléidoscope que du tableau cohérent ${ }^{3}$. Il en va de même, dans une moindre mesure, de la procession des Panathénées. Trois exemples qui concernent des problèmes posés par la frise, expliqueront mieux ce que j'entends par là. 1) Les cavaliers des parties antérieures (nord et sud, parties ouest) ne peuvent être pris pour des éphèbes - au sens habituel du mot - que si l'on oublie que ces figures datent du Ve siècle et, qu'en plus, on ne prenne pas garde aux grandes difficultés historiques auxquelles on s'expose s'agissant de l'historicité d'un entraînement à la cavalerie des éphèbes (ou d'une partie des éphèbes) avant la guerre du

3 Wedding Rites in Ancient Greece, in Opuscula Selecta, III, Lund, p. 243. 
Péloponnèse... 2) On s'étonne de n'y point trouver le navire panathénaïque et on en donne diverses raisons, la plus communément avancée consistant à argumenter du fait qu'il était remisé dans la cour de l'Éleusinion (ou du Pythion) sur la pente nord de l'Acropole, avant que la procession ne gagne le plateau rocheux. Ce à quoi d'autres répondent que la frise ne représente pas la procession sur l'Acropole, mais dans son développement complet (ce à quoi je souscris): la remise du péplos sur l'Acropole sur la partie orientale est « contemporaine » de la cavalcade de la face ouest dans la ville basse. Mais, la question toute simple qui vient à l'esprit est : le navire existait-il au Ve siècle ${ }^{4}$ ? 3) Enfin, et inversement, où sont les cités alliées, où sont les colonies, où sont les dèmes, les uns avec leurs vaches ${ }^{5}$, les autres avec vaches et panoplies?

On peut poser que tout festival religieux est composé d'éléments simples aux fonctions de base identiques et à caractère rituel. Ces éléments sont les phases successives d'un gestuel qui s'ordonne en fonction du point culminant de la fête : le sacrifice. Ces actes constitutifs de la dévotion amplifient et dramatisent les démarches simples qui l'encadrent : à savoir le simple fait de s'y rendre - la procession - et celui, ensuite, de consommer une partie de la viande - le repas sacrificiel. C'est donc précisément de la nature et de la composition du/des sacrifice(s) que dépendent en grande partie la composition et l'ambiance de la procession. Il s'ensuit un impératif méthodologique : l'étude de la procession passe par celle du sacrifice. C'est dans ce sens que nous allons prendre les choses.

\section{Le décret $I G, \mathrm{II}^{2}, 334$ et le programme sacrificiel des Panathénées}

Nos renseignements proviennent de quelques notices lexicographiques, de la frise et, surtout, d'une loi sacrée du IVe siècle $\left(I G, \mathrm{II}^{2}, 334\right)$ qui concerne les petites Panathénées. C'est à ce texte fondamental, très souvent cité, moins souvent étudié, que je vais consacrer l'essentiel de cette étude, considérant que sa célébrité l'a, en quelque sorte, desservi, et qu'il contient des leçons qui n'ont pas toutes été entendues. J'avais déjà eu l'occasion d'en donner une interpré-

\footnotetext{
4 Judicieuses remarques de N.J. NORMAN, in $A r C b N, 12$ (1983), p. $41-46$ sur la répartition des références au navire panathénaïque : aucune avant 296 , elles sont nombreuses après le décret en l'honneur du poète Philippidès. Le rite trouverait son origine dans un don fait par Démétrios Poliorcète à la cité (?).

5 Cf. infra, p. 52, sur le choix de ce mot au lieu et place de la traduction habituelle (et inattentive) par bouf.
} 
tation $^{6}$, je la reprends ici $^{7}$ en corrigeant certains points et en en développant d'autres.

Le décret est postétieur d'environ un siècle à la frise et il concerne les petites Panathénées ${ }^{8}$, alors que l'atelier de Phidias a sculpté une image des grandes Panathénées?. Il faudra tenir compte de ces différences au moment de l'interprétation. Relisons donc de nouveau ce décret ${ }^{10}$ en examinant plus particulièrement ce qui a trait aux sacrifices et à la pompè.

6 La fllle d'Atbènes, Paris, 1987, p. 36-38. J'y suis revenu dans Fêtes grecques : périodicité et inttiations. Hyakintbies et Panathénées, in A. MOREAU (éd.), L'initiation. Actes du colloque international de Montpellier; t. I, Les rites d'adolescence et les mystères, SEMA, Univ. de Montpellier, 1992, p. 19-38, surtout p. 34 sq.

7 La seule étude d'importance sur ce texte qu'il convient de relever depuis est celle de V.J. ROSIVACH, IG $2^{2} 334$ and panathenaic Hekatomb, in $P P, 261$ (1991), p. 430-442, qui entreprend de démontrer que le second complexe sacrificiel est une création du présent décret et non l'hécatombe, et que celle-ci, en revanche, doit être reconnue dans le complexe sacrificiel du premier groupe des lignes 9-10. Je me trouve en complet accord avec V.J. Rosivach sur de nombreux points : l'opposition des deux complexes sacrificiels, l'abandon de la restitution 'Ap[eí $\omega$ 1

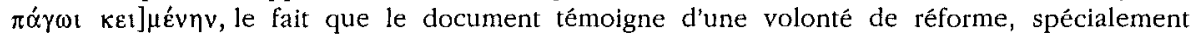
marquée pour les sacrifices du second groupe. Mais je ne pense pas comme lui que si l'on doit reconnaitre une hécatombe dans l'un des sacrifices ce soit celui de l'ancien temple qui en fournisse la matière. Pour deux raisons simples : la divinité à laquelle elle doit être adressée et le lieu où elle doit avoir lieu. Athéna Polias (préalablement appelée simplement Athéna 1. 20) est la destinatrice désignée en toutes lettres par le décret du sacrifice du grand nombre de vaches (1. 22 : Athenai tèl] Poliadl); l'endroit, c'est le grand autel d'Athéna que montrent tous les bons plans de l'Acropole et qui existe au IVe siècle, clairement identifiable dans le texte (1. 19-20 et 29: tôt bômôt ... tôt megalôi; tou bômou tou megalou). Je ne vois pas comment on peut sortir de l'enchaînement des relations : sacrifice en plein air - sur le plus grand autel de l'Acropole - du plus grand nombre d'animaux - pour la divinité principale - pour un maximum de destinataires de la chair de ces animaux : le dèmos tôn Athènaiôn (1. 25) sans aboutir à la notion d'hécatombe. Je ne relèverai pas systématiquement tous mes points de désaccord éventuels avec V.J. Rosivach (je compte le faire dans un autre cadre); je renvoie de nouveau à La fille d'Athènes, op. cit. (n. 6), p. 36-38, qu'il n'avait pas lu.

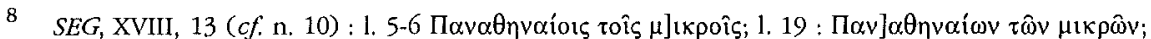

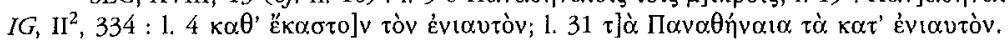

9 Si l'on en doutait, la scène de la remise du péplos l'assure (Fille d'Athènes, p. 99 sq.).

10 La partie connue depuis longtemps est le décret $I G, \mathrm{II}^{2}, 334$ (stoich. 42) (éd. et trad. F. Durrbach dans Lycurgue. Contre Léocrate, CuF, 1932, p. 13 sq.); on a découvert ensuite une loi appartenant à la même stèle (mais non jointive) et publiée par D.M. LEWIS, Law on the lesser Panathenaia, in Hesperia, 28 (1959), p. 139-149, qui datait le document de 336/5 ou 335/4 (SEG, XVIII, 13) (C.J. SCHWENK, infra, le plaçait en 336/5). Aussitôt après la publication de D.M. Lewis, L. Robert proposait de voir dans la nea qui apparait dans les deux textes des domaines affermés par la cité aux fins de fournir les ressources nécessaires au financement de la fête - plus exactement, du sacrifice -, domaines qu'il localisait à Oropos, en raison de la cession de ce territoire à Athènes par Philippe en 338-337 (Sur une loi relative aux petites Panathénées, Hellenica, XI-XII, 1960, p. 189-203). Cette identification a été depuis plusieurs fois remise en cause. Par D.M. LEWIS lui-même (dans Hesperia, 37 [1968], p. 374 n. 18 et dans Les biens publics dans la cité, in La cité grecque d'Homère à Alexandre, O. MURRAY et S. PRICE (éds), trad. franç. 1992, p. 291 sq.) et par M.K. LANGDON, publiant une décret très mutilé des tribus Aigéis et Aiantis à propos de domaines qu'elles possédaient à Oropos, et qui place la Néa (avec majuscule) dans une île du même nom au nord de l'Egée, entre Lemnos et l'Hellespont (Hesperia, 56 [1987], p. 47-58). Depuis, O. HANSEN a proposé de restituer le nom de Nikomachos à la 1.2 et de dater la loi de 341/0; dans cette configuration chronologique, l'explication de la néa par L. ROBERT serait 


\section{Le premier complexe sacrificiel}

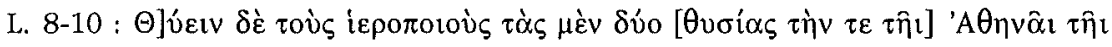

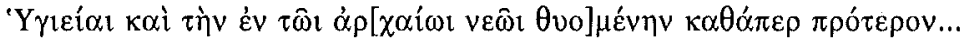

Que les hiéropes offrent, comme précédemment, deux sacrifices, le premier à Athéna Hygiéia et le second dans l'ancien temple (trad. F. Durrbach) ${ }^{11}$.

Comme on s'en apercevra plus loin, ces deux sacrifices ne sont pas les seuls auxquels le décret s'intéresse. Il sera question de deux autres sacrifices après la rupture de la ligne 17, essentielle à la compréhension du document. Nous parlerons donc plutôt de deux complexes sacrificiels successifs, le premier introduit (1. 8) par $\mu \grave{\varepsilon} v$ et le second, par $\delta \varepsilon ́$ (1. 16). Plusieurs questions se posent à propos de ce premier ensemble : pour qui sont ces sacrifices ? où ontils lieu ? et avec quelles bêtes?

1) Les divinités? Si le texte nomme la première de celles auxquelles il est consacré, il nous manque de pouvoir identifier directement l'autre. 2) Les lieux ? Le sacrifice à Athéna Hygiéia doit avoir eu lieu sur un autel à proximité de sa statue aisément localisée grâce au texte de Pausanias (I, 23, 4) qui décrit les statues que l'on rencontre en entrant sur l'Acropole et par la trouvaille in situ d'une base inscrite $\left(I G, \mathrm{II}^{2}, 395\right)$. Nous nous trouvons à la sortie des Propylées, au sud-ouest ${ }^{12}$. Mais où a lieu de second? 3) Les victimes ? Si l'origine des animaux du deuxième complexe sacrificiel est connue, les rédacteurs du décret n'ont pas jugé bon d'en parler pour ce premier complexe. Cette remarque nous fait pénétrer au cœur du problème. S'ils font le silence sur l'origine des victimes de ce premier ensemble, c'est qu'elle reste la même (kathaper proteron) et que le dèmos n'a pas à légiférer à leur propos. En revanche, les prescriptions seront d'autant plus précises, d'autant plus exhaustives, qu'elles seront nouvelles.

Une partie de nos interrogations trouverait des réponses satisfaisantes si nous pouvions être assurés de la justesse de la restitution de la ligne 10.

\footnotetext{
évidemment caduque (Eranos, 87 [1989], p. 70-72). Et l'auteur identifie la Néa à l'île d'Halonnésos, au sud de Lemnos, ce territoire aurait appartenu à Athènes et à Lemnos au Ve siècle. - Réédition récente des deux textes par Cynthia J. SCHWEnK, Atbens in the Age of Alexander. The Dated Laws $\&$ Decrees of 'the Lycourgan Era' 338 - 322 B.C., Chicago, 1985, p. 81-94, avec bibliographie antérieure. - Commentaires : outre les travaux classiques de A. MOMMSEN, L. DEUBNER et H.W. PARKE, on verra V.J. ROSIVACH qui a repris l'étude de ce document dans l'article cité à la note 7 et l'a prolongée dans un ouvrage: The System of Public Sacrifice in Fourtb-Century Atbens, Atlanta, 1994 (American Classical Studies, 34).

11 Loc. cit. n. précédente.

12 Plan de l'endroit, photo de la base in situ dans J. TRAVLOS, Bildlexikon zur Topographie des antiken Atbens, Tübingen, 1971, p. 124 et Abt. 169 et 170 (renvois à la littérature de première main).
} 


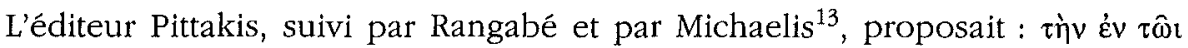

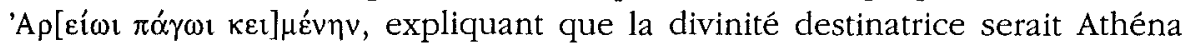
Aréia arguant du fait qu'elle disposait, aux dires de Pausanias (I, 28, 5), d'un autel sur l'Aréopage. Cette hypothèse présente de nombreuses difficultés. 1) Dans les trois autres sacrifices mentionnés, la «personnalité » d'Athéna est précisée par son épiclèse ${ }^{14}$, il serait étrange qu'on ait utilisé, spécialement pour Athéna Aréia, une telle périphrase «topographique ${ }^{15}$. Si l'on déroge au mode habituel de désignation qui consiste à nommer la divinité pour choisir à la place de circonscrire un espace (en), c'est probablement parce que cet espace était le théâtre d'une liturgie complexe qui concernait plusieurs divinités. 2) On connaît avec suffisamment de certitude le trajet de la procession, montant directement du coin sud-est de l'agora à l'Acropole par la «Rue » des Panathénées, pour juger très peu crédible un détour (à partir d'où ?) qui conduirait la procession de la sortie de l'agora à l'Aréopage avant de revenir ensuite à l'Acropole. 3) Sachant enfin que la statue d'Hygiéia se trouve sur l'Acropole au moins depuis le Ve siècle, et que le sacrifice qui lui est consacré forme, avec celui qui nous intéresse, un complexe sacrificiel (ce qui signifie en particulier qu'il s'agit d'un même ensemble de victimes), il est difficile de l'imaginer le second fort loin de lui. "A sacrifice outside the Acropolis is completly out of context here " va jusqu'à dire C. J. Herington ${ }^{16}$, et je dois dire que cela me semble la voix du bon sens.

On a proposé une autre restitution qui, comme la première remplit exactement l'espace manquant des douze lettres, une restitution qui place le

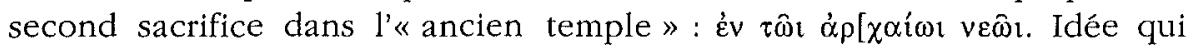
remonte à J.L. Ussing ${ }^{17}$, reprise par A. Mommsen ${ }^{18}$, puis suivie, entre autres, par E. Pfühl19, W. Dittenberger ${ }^{20}$, puis par L. Deubner ${ }^{21}$, pour devenir depuis l'opinion commune (ainsi $I G \mathrm{II}^{2}$ ). Sacrifier dans l'« ancien temple », c'est, si l'on se conforme à la phraséologie en usage au IVe siècle, sacrifier dans ce que les

13 Ephèmeris Arcbaiologikè, 4 (1882), p. 608-610, $\mathrm{n}^{\circ}$ 1064; Antiquités belléniques, II, Athènes, 1855, p. 439-44, $\mathrm{n}^{\circ}$ 841; Der Partbenon, Leipzig, 1881, Anbang 2, Die Panatbenden, 322 (fait suivre la mention d'Athéna Aréia d'un point d'interrogation).

14 Si elle est omise 1. 20 pour la Polias, elle est en revanche bien précisée plus loin, 1. 22 :

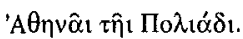

15 Quand il s'agit de désigner Athéna Aréia, les documents épigraphiques du IVe siècle le font de façon naturelle (j'entends comme pour toute autre divinité); en témoigne, par exemple, la version acharnienne du serment des éphèbes.

16 Athena Parthenos and Athena Polias, Manchester Univ. Press, 1955, p. 31 n. 4.

17 Inscriptiones Graecae ineditae, Havniae, 1847, p. 45-50, $\mathrm{n}^{\circ} 54$.

18 Heortologie, Leipzig, 1864, p. 174, puis dans Feste der Stadt Atbens im Altertum, Leipzig, 1898, p. 118-120

19 De Atbeniensium pompis sacris, Diss., Berlin, 1900, p. 15-16.

20 Syll. ${ }^{3}, 271$. Dans les versions précédentes de la Sylloge, W. Dittenberger avait préféré la restitution de Pittakis.

21 Attiscbe Feste, Berlin, 1932 (réimpr. Hildesheim, 1959), p. 26 n. 2. 
modernes ont coutume d'appeler l'Érechthéion ${ }^{22}$, c'est-à-dire, s'agissant d'Athéna, sacrifier à Athéna Polias ${ }^{23}$. Quoi de plus attendu qu'une telle conclusion s'agissant de la divinité qui, aux grandes Panathénées, reçoit le fameux peplos, celle à laquelle l'ensemble festif est dédié. Mais cette théorie n'est pas non plus sans difficultés. Comme le disait W. Dittenberger ${ }^{24}$, on ne sacrifie pas les animaux dans les temples, on sacrifie à l'extérieur (V.J. Rosivach le rappelle $e^{25}$.

L. Ziehen avait déjà été sensible à cette question de liturgie, ce qui l'avait amené à proposer une restitution qui autorise l'exécution d'un sacrifice sanglant

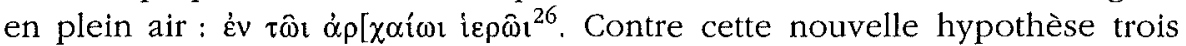
objections se présentent. La première n'est pas dirimante : il faudrait admettre l'existence de l'expression archeion bieron, alors qu'on ne la rencontre pas ailleurs; la seconde pose le problème de l'identification : sur quel(s) autel(s) sacrifierait-on dans cette hypothèse et dans quel sanctuaire? On pourrait penser au temenos de Pandrosos en raison de la loi liturgique transmise par Philochore: qui sacrifie un bœuf à Athéna doit sacrifier une brebis à Pandrosos ${ }^{27}$, mais le seul autel qu'on y connaisse n'a rien à voir avec Athéna : c'est un autel de Zeus Ktésios... Mais il est bien entendu possible qu'il en existât un autre ${ }^{28}$. Enfin il

22 On marche toujours sur des oufs dès lors qu'il s'agit de topographie acropolitaine, particulièrement à propos du bâtiment appelé Érechthéion. Les arguments de K. JEPPESEN pour le débaptiser et qui font douter que ce qu'on a coutume d'appeler la maison des arrhéphores fut effectivement une "maison », ces arguments sont forts et doivent toujours rester en mémoire : Where was the so-called Erecbtheion?, in AJA, 83 (1979), p. 381-394 et Further Inquiries on the Location of the Erechtheion and its Relationship to the Temple of the Polias. 1. Prostomaion and Prostomion, in $A J A, 87$ (1983), p. 325-333 et The Theory of the Alternative Erechtbeion, Arhus, 1987 (Acta Jutlandica LXIII : 1).

23 Pour la démonstration, qui utilise le témoignage essentiel des inventaires qui débutent en 371 (IG, II ${ }^{2}, 1421$ et sq.) du Parthénon, de la Chalkothèque et de l'archaios néôs, je renvoie à l'ouvrage de C.J. HERINGTON cité n. 16. Il faut pourtant se montrer prudent et laisser ouverte l'hypothèse (évoquée par $\mathrm{E}$. Voutiras dans une conversation lors du colloque) où les Athéniens de cette époque pourraient avoir encore en tête l'ancien temple d'Athéna - le Dinsmoor -, ce qui conviendrait à la nécessité de se trouver en plein air ( $c f$. infra); mais sur quels autels?

$245 y / l^{3}, 634$.

25 Art.cit. (n. 7), p. 440 n. 35.

26 Die panatbenaischen und eleusiniscben Hieropoiol, in $R b M(1896)$, p. 93 et $R E$, XVIII, col. 471. Il faut noter que la restitution de Ziehen est trop longue d'une lettre, mais, selon C.J. SCHWENK (cité n. 10, p. 93), la ligne précédente aurait compté aussi 43 lettres. S. HuMPHREYS rejette elle aussi

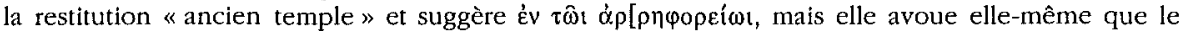
"terme arrbepboreion n'est pas attesté comme le nom d'un espace autour de la maison des arrhéphores »; alors? (Lycurguls of Butadae. An Atbenian Aristocrat, in J.W. EADIE et J. OBER [éds], The Craft of The Ancient Historian. Studies C.G. Starr; Lanham, MD, 1985, p. 228-229). Faut-il discuter la restitution de Fr. SOKOLOWSKI, Lots sacrées des cités grecques, Paris, $1969, \mathrm{n}^{\circ} 33$, qui écrit

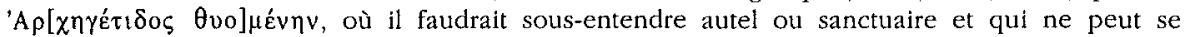
recommander d'aucun lieu connu portant ce nom sur l'Acropole?

$27 \quad F G r H, 328$ F 10.

28 M. KORRES évoque (p. 178) les récentes découvertes de A. Papanikolaou qui sont en rapport avec ces questions : une petite stoa ionique de Pandrosos ainsi que les traces d'un enclos défensif 
reste une difficulté essentielle, d'un tout autre ordre, la restitution de Ziehen est trop longue d'une lettre, alors que le texte est gravé stoichedon. Mais C.J. Schwenk ${ }^{29}$ signale d'autres écarts avec la règle du stoichedon...

De ce raisonnement, on le constate aisément, il est difficile de sortir. C'est, je pense, parce que l'on imagine toujours que ce(s) sacrifice(s) qui nous manque(nt) s'adressai(en)t à la seule Athéna (Polias ou une autre). C'est ainsi que l'on voit certains commentateurs renoncer à la restitution de l'« ancien temple » parce qu'un sacrifice olympien ne pourrait se dérouler, au IVe siècle, dans un naos. La formulation, d'ailleurs, invite à abandonner cette conception; s'il s'était agi de la seule Athéna Polias, on aurait eu une formule répondant à

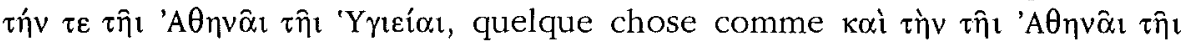

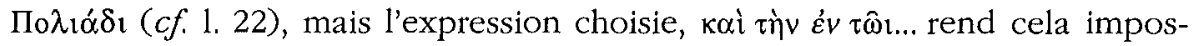
sible. Dans la mesure même où une telle désignation est inusitée, il me semble légitime de penser à une série de sacrifices pour diverses divinités. Alors à qui ? Peut-on se proposer de les identifier?

Je propose d'ajouter Érechthée à la Polias. Je ne veux pas reprendre ici complètement une démonstration que j'ai faite ailleurs ${ }^{30}$. Je veux seulement évoquer les trois textes sur lesquels je me suis appuyé : 1) Un passage, peutêtre interpolé, de l'Iliade (II, 546) où les kouroi athéniens offrent à Érechthée, "l'enfant de la glèbe féconde " des taureaux et des agneaux à chaque retour de l'année » (les lecteurs d'A. Brelich ne peuvent manquer de se souvenir de son analyse de la fête comme cérémonie de capo d'anno ${ }^{31}$ ). 2) L'histoire, rapportée par Hérodote, où les Épidauriens, obligés, pour façonner des statues en bois d'olivier, comme le leur recommande la Pythie, de s'adresser aux Athéniens pour obtenir de couper chez eux les arbres nécessaires; les Athéniens acquiescent «à condition qu'ils amèneraient chaque année des victimes à Athéna Polias et Érechthée » (V, 82). 3) Enfin, un passage récemment connu de l'Érechthée d'Euripide où Athéna, après la mort d'Érechthée, enjoint à Praxithéa d'accomplir un certain nombre d'actes religieux qui sont des fondations : «qu'on lui construise un sanctuaire au milieu de la ville, avec une enceinte de pierre... ». C'est sous le nom de Poséidon Érechthée que «les citoyens l'invoqueront quand ils lui immoleront des boufs » ${ }^{32}$. Je considère que dans ces trois témoignages la fête en question, ce sont les Panathénées.

V.J. Rosivach semble assuré que dans ce que nous appelons l'Érechthéion, tous les autels recevaient des sacrifices non-sanglants ${ }^{33}$; cela ruinerait l'hypo-

rapidement construit au N-O de l'Érechthéion avant l'attaque perse de 480 (R. ECONOMAKIS [éd.], Acropolis Restauration. The CCAM Interventions, Londres, 1994).

29 Op.cit. (n. 10), p. 93.

30

Art. cit. (n. 6), p. 22-26.

31 Paides e parthenoi, Rome, 1969, p. 314-347.

32 C. AuSTIN, Nova Fragmenta Euripidea in Papyris reperta, Berlin, 1968, p. 15 sq. et 22 sq., col. $7,90 \mathrm{sq}$.

33 Art.cit. (n. 7), p. 440-441, n. 35. 
thèse que je suis en train de formuler, puisqu'à l'issue de ce premier enchaînement de sacrifices, on procédait à une première distribution de viande; il fallait donc que les sacrifices qui le composaient fussent olympiens. Je ne vois pas de raison pour que les autels de Poséidon Érechthée, de Boutès et d'Héphaïstos que cite Pausanias (I, 26, 5) soient appréciés, de ce point de vue, de la même façon. Laissons le dernier de côté (d'ailleurs évidemment ouranien), si les rituels dédiés à Boutès étaient probablement plutôt chthoniens, je doute de la nature héroïque du premier. Je rappelle l'étonnement dont fait preuve Cicéron lorsqu'il apprend de la bouche des Athéniens qu'Érechthée est traité comme un dieu (de nat. deorum, III, 49). D'une façon génétale, l'utilisation que nous faisons de l'opposition sacrifice sanglant - sacrifice chthonien, parce que trop rigoureuse, est trop simpliste; et nous savons par de nombreux exemples que la liturgie emprunte souvent des caractères soi-disant spécifiques à l'une ou l'autre des grandes catégories. C'est particulièrement vrai pour le culte des héros qui utilise de temps à autre (voir Thésée à Athènes) des formes classées souvent comme ouraniennes. Par ailleurs l'architecture hétérodoxe et complexe du bâtiment appelé Érechthéion, avec ses portiques, invite à imaginer une scène analogue à celle que montre la fameuse hydrie à figures noires de Berlin ${ }^{34}$ avec son Athéna assise, l'autel à volutes où brûle le feu sacrificiel, le serpent, l'olivier au second plan, le bouf dans ou devant le temple dorique.

On me pardonnera de ne pas convoquer ici en outre tous les documents qui présentent Érichthonios et/ou Érechthée comme synnaos(i) de la déesse dans son épiclèse de Poliade, et cela, de la même façon que le font les textes de l'Iliade et d'Hérodote qui associent Athéna (Polias) à Érechthée ${ }^{35}$.

Comment conclure malgré les incertitudes? Loin d'être assurée, la restitution habituelle de l'ancien temple a néanmoins pour elle plus d'arguments. Le lieu? On échappe difficilement au bâtiment appelé traditionnellement Érechthéion et à son environnement immédiat ${ }^{36}$, sur divers autels, intérieurs et extérieurs. Les divinités : la Polias, et deux divinités que les sources lui associent séparément : Érechthée-Érichthonios et Pandrosos (la Cécropide fidèle et obéissante, si souvent associée à Athéna Polias dans les dédicaces hellénistiques et impériales des parents d'arrhéphores, est honorée par l'epiboion, conjointement à la déesse ${ }^{37}$ ).

34 L.R. FARNELL, The Cults of the Greek States, Oxford, 1909, 1, pl. 14 b, face p. 325.

35 À propos du dédoublement d'Érichthonios et d'Érechthée, voir mon étude sur La liste des premiers rois d'Atbènes dans la Bibliothèque d'Apollodore. Histolre et politique, à paraître dans Polkilia, 1996.

36 Bente KIILERICH fournit, sur cet environnement, d'utiles mises au point dans une étude qui porte sur l'époque archaïque: The Olive-Tree Pediment and the Daugbters of Kekrops, in $A A A H$, VII, 1989, p. 1-21, surtout p. 11 sq.

37 Je n'ai rien à retrancher de ce point de vue à ce que j'ai développé dans La fille d'Athènes, p. 36-38. 
Et, peut-être aussi, Boutès et même Héphaïstos. Le premier en raison de son lien avec le génos qui préside à la fois aux rites pour la Polias (prêtresse), et aux rites pour Poséidon Érechthée (prêtre) : les Étéoboutades. Le second pour ses liens connus avec la Fille de Zeus à travers l'un des mythes de la naissance d'Érichthonios (à propos duquel il est bon de rappeler que la tradition place son tombeau dans le temple de la déesse) et à travers une intime parenté de fonctions. Mais l'hypothèse n'en est pas nécessaire ${ }^{38}$.

Kathaper proteron... Pour ce premier complexe sacrificiel, les hiéropes procèderont comme par le passé. En matière théologique, cette précision, ou plutôt ce rappel, n'offre pas grand intérêt : on sait bien que le décret n'a pas pour objet de changer les divinités honorées lors de la fête; en revanche, cette référence au modèle ancien que doivent suivre les hiéropes nous importe pour ce qui concerne la dernière question que nous avons posée plus haut : quelles bêtes sont immolées dans les sacrifices de cette première série?

Ignorants que nous sommes des éventuelles modifications au programme de la fête, nous ne pouvons éviter de nous tourner vers l'amont, vers le seul document qui nous renseigne sur le passé de la fête : la frise du Parthénon. Sur les frises sud et nord, qui décrivent la marche de la procession vers la scène centrale de la façade est où se déroule la remise du péplos, à proximité de cette dernière, sont représentés les animaux de sacrifice : au sud, ce sont des boufs, en aussi grand nombre que le permet l'impératif de symétrie entre les deux faces (plus d'une douzaine), au nord, ce sont quatre boufs et quatre moutons ${ }^{39}$. Il est temps alors de rappeler l'hypothèse émise il y a près d'un siècle par E. Pfühl : les animaux de la frise nord sont offerts par les quatre anciennes tribus ioniennes. L. Deubner est fort convaincant lorsqu'il identifie cet ensemble de victimes séparé de la frise sud comme celui qui alimente le complexe de l'« ancien temple», et qu'il voit dans la «foule » de ceux de la frise nord l'ensemble concerné par l'hécatombe ${ }^{40}$. Dans cette hypothèse, les dispositions de notre inscription pourraient être étendues au Ve siècle et aux grandes Panathénées.

38 C'est la présence obligée des Étéoboutades dans ces circonstances, à l'époque classique, quí m'y fait penser : ils détiennent la prêtrise de la Polias et celle de Poséidon Érechthée, d'où une possible association avec un sacrifice au frère d'Érechthée, l'éponyme du génos.

39 Pour apprécier la différence il faut savoir qu'hormis cette partie, les deux côtés principaux de la frise se correspondent absolument: de l'ouest vers l'est, cavaliers, apobates et thallophores occupent des plaques symétriques. Dans le dernier tiers environ alors que, sur le côté nord (côté Erechthéion), se succèdent dans l'ordre les citharistes, les aulètes, les hydriaphores, les skaphéphores, les moutons puis les bœufs, sur le côté sud, les bœufs occupent tout l'espace en compagnie des victimaires.

40 op. cit. (n. 21), p. 26-27; HeRINGTON, op. cit. (n. 12), p. 32; Dans La fille d'Athènes, p. 37 sq., je lie le couple bovin-ovin suggéré par Pfühl à la loi liturgique de l'épibolon donnée par Philochore. 


\section{Première distribution de viande}

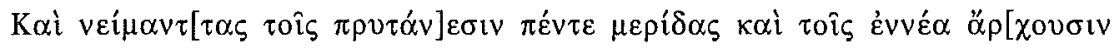

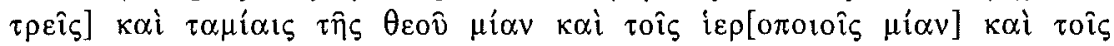

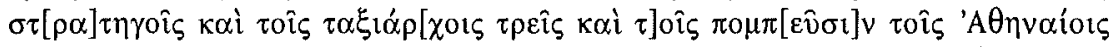

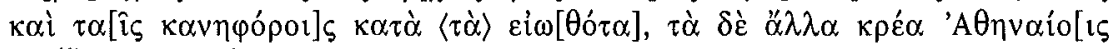
$\mu \varepsilon p i \zeta \varepsilon ı v$ (1. 10-16).

Et qu'après avoir distribué aux prytanes cinq parts de viandes, trois aux neuf archontes, une aux trésoriers de la déesse, une (?) aux hiéropes, trois aux stratèges et aux taxiarques, et les portions d'usage aux Athéniens qui ont fait partie du cortège ainsi qu'aux canéphores, ils distribueront le reste de la viande aux Athéniens (d'après F. Durrbach).

Laissant de côté les discussions que l'on pourrait mener sur certaines restitutions de nombre de parts de viande ${ }^{41}$, deux questions me semblent devoir être posées: l'une à propos des canéphores et l'autre à propos des «Athéniens qui ont fait partie du cortège».

\section{Canéphores}

On voit combien la restitution tient à peu de chose : l'alpba de l'article ! Certes, on sait bien que les canéphores appartiennent à la procession; un regard jeté sur la frise les découvre en tête des participants côté sud, comme côté nord, si bien qu'elles seules, avec les ergastines, "débordent » sur le côté est. Et, puisqu'il fallait trouver des participantes à la procession on pouvait en théorie hésiter entre les arrhéphores, les ergastines et les skaphéphores ${ }^{42}$. Mais, le service le plus prestigieux et le plus caractéristique, c'est, évidemment, celui de canéphore. La restitution a donc pour elle la plus grande vraisemblance malgré son caractère aventureux eu égard aux seuls arguments épigraphiques $^{43}$.

41 Ennea plutôt que treis pour les archontes? Rangabé restitue plutôt mian pour stratèges et taxiarques, mais le mot remplit moins bien la lacune que treis... Il me semble néanmoins apercevoir une règle : on attribue une part de viande par collège de magistrats à représentation tribale. C'est vrai pour les hiéropes, les trésoriers, cela le serait si l'on suivait Rangabé pour les stratèges et les taxiarques; c'est la même quantité qui est donnée aux prytanes : 5 parts pour 50 , soit une part pour dix. Les archontes seraient traités différemment, et de façon évidemment anachronique.

42 Les hydriaphores sont, aux Panathénées, des hommes (frise nord, plaque VI).

43 Sans qu'il s'agisse d'un argument pour cette restitution, il faut rappeler ce passage de la loi $I G, \mathrm{II}^{2}, 333$, fr. $c, e, f, 1.5$ où, parmi des dispositions prises à l'instigation de Lycurgue pour la célébration des grandes Panathénées, il est question d'objets destinés aux canéphores (ou, plus exactement, à la canéphorie), ce que confirme le décret honorifique de Stratoclès annexé à la Vie de l'orateur par le PS.-PLUTARQUE : « il fit confectionner pour la déesse du matériel de culte, à savoir des Victoires en or massif, des vases de procession en or et en argent et des ustensiles d'or pour cent canéphores " (852b). Sur la signification du chiffre cent et son rapport avec la 
Une autre question, qu'on est étonné de n'avoir pas trouvée posée ailleurs : comment expliquer que des parthenoi reçoivent ès-qualités des parts kata ta eiôtbota dans un sacrifice politique, voire dans un sacrifice tout court? Problème apparemment mineur, annexe, par rapport au programme que je me suis fixé, mais qu'il convient d'aborder dans la mesure où la réflexion sur cette question permet d'avancer dans l'interprétation du système sacrificiel que décrit l'inscription. La réponse ne va pas de soi et on ne se débarrassera pas de cette étrangeté en disant qu'elle n'est pas unique, que l'on trouve quelques autres exemples d'une telle pratique. Il restera qu'il convient d'en comprendre la raison, aux plans religieux (liturgique), sociologique et politique.

Les filles reçoivent, dans ce contexte particulier, des parts de viande. Des femmes n'en recevraient pas. C'est en d'autres lieux, pour d'autres divinités, excluant généralement la présence des hommes, que les femmes sacrifient en corps. Des femmes peuvent, évidemment, participer au repas sacrificiel qui mettra un terme à la fête, au Céramique dans le bâtiment prévu à cet usage, et cela sur la part reçue par leur époux. Mais ce n'est pas de cela qu'il s'agit ici puisqu'en ces circonstances (lieu et temps), l'accès au partage est signe d'appartenance à la communauté politique, désignant les bénéficiaires comme citoyens. Il y a donc bien là une singularité.

Pour interpréter cet usage singulier et pour retrouver aussi, derrière la complexité de tous les sacrifices (au moins quatre) qu'évoque le décret, une conception d'ensemble, une signification globale, il est maintenant nécessaire de prendre un peu de hauteur avec le document et de considérer les phases principales du texte. Il s'articule en deux grandes parties : la première s'ouvre, on l'a dit, par le $\mu$ غ̀v de la ligne 8 auquel répond, pour la seconde, le $\delta \dot{\varepsilon}$ de la ligne 16. Il existe d'autres ruptures. À l'échelon inférieur, les obligations des

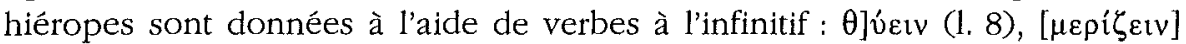

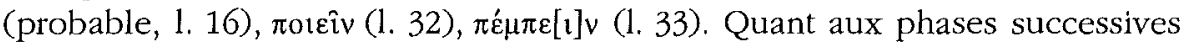
du rituel, elles sont scandées par des participes qui marquent qu'une action

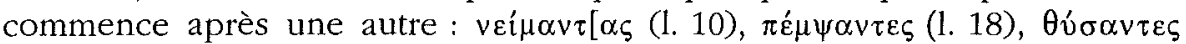
(1. 21). Tout cela ne manque pas de rigueur et ces charnières dans le texte justifient et illustrent clairement notre analyse qui identifie deux complexes sacrificiels (associant deux ou plusieurs divinités, auxquelles sont immolés deux groupes différents d'animaux), lors desquels il est procédé à deux distributions de viandes avec deux modes de partage. C'est dans ce contexte que le neimantas de la 1, 10 répond au thuein de la 1. 8. Dans cette logique du «récit», un point ne manque pas d'attirer l'attention: le pempsantes tèn pompèn de la 1. 18, qui appartient à la seconde série. «Après avoir processionné... » : cela signifie que la procession n'est pas terminée quand a lieu la première série de sacrifices, la première distribution de viandes. Certes, il n'y a pas loin de l'«ancien sanctuaire » tel que nous l'avons identifié plus haut à 
l'autre grand rendez-vous sacrificiel qui le bômos bo megalos: le grand autel d'Athéna ${ }^{44}$, mais l'expression employée prouve que la pompè n'est considérée comme terminée qu'une fois que l'on a atteint le grand autel.

Une fois cette perspective fermement tracée, oublions maintenant le détail pour considérer le tout. Vu de plus haut, je crois que l'on peut proposer une perspective simplifiée qui ne retiendrait de cet ensemble complexe qu'un seul et même sacrifice à Athéna Polias. Il se déroule en deux parties : dans l'« ancien temple », puis sur son grand autel. Les sacrifices à l'Hygieia comme à la Niké ne sont que des appendices, des amplifications, des greffons qui ont eu la chance de prendre. Nous en connaissons même les circonstances historiques, récentes au regard de la très longue histoire d'une telle fête. Mais l'essentiel, c'est la Polias. Dans cette perspective globalisante, et à considérer les deux distributions de viandes, on n'échappe à l'idée que celle de l'« ancien sanctuaire » revêt, par son caractère préliminaire, par l'identité de ses destinataires, le caractère d'un géras. Il convient certes d'apprécier à sa juste valeur, relative, - de la démocratie relative de la fin du IVe siècle l'importance et la dignité de tel ou tel collège de magistrats cité ici, mais l'ensemble des participants n'en constitue pas moins une élite, au moins un choix d'individus qui prennent la place de ceux que dans l'épopée ou, même, dans le sacrifice privé, on cherche à honorer (les rois, le héros, l'hôte). La cité distingue ainsi une partie de ses citoyens pour s'honorer elle-même. La différence avec la pratique sacrificielle habituelle, bien analysée par J.-L. Durand $^{45}$, c'est que l'on n'a pas affaire ici au corps d'une bête, mais à toute une collection. Mais ce n'est qu'une question d'échelle, la conception générale ne change pas. S'agissant d'opérer sur un seul animal, le procédé le plus fréquent consiste à faire de lui des parts égales, une fois le prélèvement d'honneur effectué. S'agissant d'un nombre important d'animaux, c'est à partir d'un groupe particulier, que sera constituée la part du privilège, alors que les animaux restants donneront lieu au partage égalitaire. C'est la principale différence entre les sacrifices de l'«ancien temple » et celui du grand autel. Au total, tout se passe donc comme si c'était une seule et même bête qui était sacrifiée à la Polias, et dont le corps serait soumis au traitement habituel, mais en deux lieux, successivement.

Si cette conception correspond bien à l'arrière-plan du sacrifice panathénaïque annuel, alors la cité ne distribue pas une part banale aux filles qui portent-la-corbeille, elle leur assigne une part de geras. Loin de minimiser la portée de cette distribution de viande aux filles, notre interprétation ne lui en attribue que plus de valeur symbolique. Les raisons sont de plusieurs ordres et

44 L'identification du bômos bo megalos (cf. 1. 29 tou bomou tou megalou) de l'inscription avec le grand autel $(15 \mathrm{~m}$. de largeur env.) à degrés reconnu par les archéologues entre l'Érechthéion et le Parthénon, ainsi que celle d'Athéna Polias comme destinatrice de celui-ci ne font aucun doute (pour le dernier point, comparer les 1. 20 et 22).

45 Bêtes grecques, in M. DETIENNE, J.-P. VERnANT, La cuisine du sacriflce en pays grec, Paris, 1979 , p. $133-166$, surtout p. 155 sq. 
nous ne pouvons en détailler ici toutes les composantes ${ }^{46}$ : elles sont religieuses parce qu'elles ont à voir avec la pureté des vierges, avec la ritualisation du passage d'âge et elles sont sociales par cette ostentation du groupe des belles filles à marier. Mais on sait bien aussi qu'ainsi, ce sont surtout leurs pères que la cité honore.

\section{«Les Athéniens ayant fait partie du cortège »}

Toutes les viandes de la première série ne sont pas évoquées de la même façon. Il est question d'abord de la distribution de parts en nombre variable à différents collèges de magistrats (neimantas...), puis le texte évoque les «Athéniens qui font partie de la pompè » et les canéphores. Mais, contrairement à ce qui se passe pour les magistrats où le nombre de parts de viande est réglementée pour chaque collège, il n'est donné aucune indication quantitative aussi bien pour les canéphores que pour les « Athéniens ayant fait partie du cortège ». Cette première série se termine par une dernière précision : " et le reste de la viande (alla krea) sera distribué aux Athéniens". Tout cela ne va pas de soi. Pourquoi cette différence de traitement? Que représente-t-elle? Qui sont les «Athéniens qui font partie du cortège »? En quoi sont-ils différents des «Athéniens» qui reçoivent le reste de la viande, et qui sont ces derniers?

On peut aborder le problème par la dernière question. Je suis sensible à un changement dans la façon de désigner la viande des bêtes immolées : on est passé d'une opération de partage (neimantas ... pente ... méridas), de découpe, à un collectif, une richesse indivise : kréa, de la viande. J'interprète cette différence de la façon suivante : après qu'auront été servis les magistrats et aussi les pompeusin tois Athènaiois et les canéphores, tout ce qui restera de viande (non encore mesurée, découpée), sera distribué « aux Athéniens »; cela signifie que ces chairs iront rejoindre la masse de cette autre viande qui provient des achats des hiéropes, comme nous allons le voir bientôt, et ne s'en distinguera plus; ce reliquat subira un mode de découpe tout à fait différent de celui qui a été nécessaire pour isoler les parts du sacrifice d'honneur. Quant aux «Athéniens », distincts de ceux que la cité honore et de ceux qui "participent au cortège », ce sont ceux dont il est question dans la seconde partie du texte, le « dèmos des Athéniens » (1. 24), réparti par dème.

En remontant dans le texte, et ainsi dans les opérations successives sur les animaux, il reste à identifier les «Athéniens qui font partie du cortège », qui, donc, ne sont pas confondus avec «les Athéniens» ou «le peuple des Athéniens ». Il n'y a malheureusement que la frise et les sources littéraires pour les identifier. Et elles répondent : skaphéphores, hydriaphores, thallophores...; elles répondent : les porteurs de biéra, d'objets nécessaires à la liturgie; elles répondent : les jeunes filles - ergastines et canéphores. Autant de participants

46 Je renvoie au chapitre «La fille sur la place ou porter-la-corbeille », in La fille d'Athènes, p. 287-335. 
qui ne sont pas rassemblés par dème ou en d'autres groupes politiques, mais par âge et pas sexe, en groupes qui sont des vestiges, pour certains, du système des classes d'âge. La représentation de la frise confirme, en gros, les sources textuelles, essentiellement lexicographiques, quil nous renseignent à propos du rôle liturgique et des conditions d'accès à ces groupes. Dans la pompè, ils sont mis en évidence, ce sont ceux qui forment la première partie de la procession et qui reçoivent la viande de la première partie du programme sacrificiel panathénaïque.

Les Athéniens ne ressentent pas la nécessité de se décrire à eux-mêmes le contenu de leur fête. Pour que le message soit clair il suffit, pour tout ce à quoi le décret ne touche pas, de renvoyer aux pratiques habituelles, ancestrales. En revanche, la précision est indispensable dès lors qu'il s'agit d'innover. Je ne vois donc pas pourquoi on aurait jugé utile de donner cette longue liste des parts à distribuer aux diverses catégories de magistrats s'il ne s'agissait pas de la modifier. J'en déduis que les «portions d'usage » ne valent que pour les canéphores et les autres catégories de processionnaires. Pour les magistrats, le dèmos propose, pour quelque raison que ce soit, et qui nous échappe, une nouvelle évaluation ${ }^{47}$ et une nouvelle répartition de ces parts d'honneur. Une hypothèse alors : ne serait-ce pas pour permettre de se ménager un reliquat, $t a$ alla kréa, et pour augmenter ainsi le volume à distribuer au peuple ? $^{48}$

C'est par deux fois que, dans cette partie du document, on se réfère à la coutume : katbaper proteron, kata <ta> eiôtbota. Dans cette même partie, et à l'inverse de ce que l'on trouve quelques lignes plus loin, il n'est pas fait mention de l'origine des animaux de sacrifice. La meilleure explication de ce contraste réside, à mon sens, dans le fait que, sur ce point, le décret n'apporte rien de nouveau. Inutile, pour la boulè et l'ecclésia de revenir sur ce que tout le monde sait. Les bêtes qui servent à fournir les parts d'honneur aux participants à la première partie de la procession (les magistrats, les groupes définis par leur âge et leur sexe), on continuera à se les procurer et à les choisir « comme avant ", "selon la coutume ». Il est très tentant évidemment de suivre la suggestion de Pfühl suivie par Deubner, et que j'ai moi-même reprise ${ }^{49}$, qui voit dans les quatre bœufs et quatre moutons de la frise nord l'offrande faite par les quatre tribus pré-clisthéniennes.

47 Le total sera automatiquement affecté par une réforme si l'on considère comme moi que le texte n'a de sens que si la meris a une valeur fixe.

48 Cette interprétation présente néanmoins une difficulté : on s'attendrait à ce que la construction de la phrase marque une opposition plus significative entre ses parties. Or nous lisons : kat neimantlas tols prytanjesin pente meridas... lkat tjois pompeusin tois Atbènaiois... Le $k a t$, restitué, ne remplit pas bien ce rôle.

49 Travaux cités aux n. 19 et 21; La fille d'Athènes, p. 37 


\section{Second complexe sacrificiel, seconde distribution de viande}

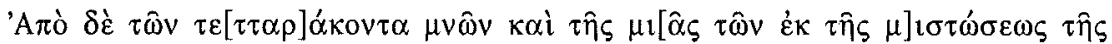

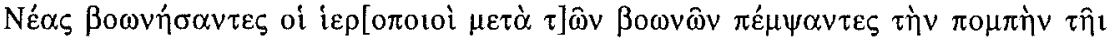

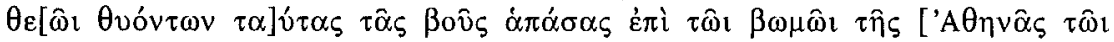

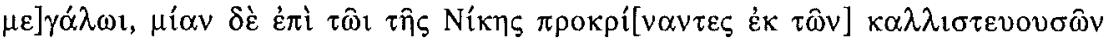

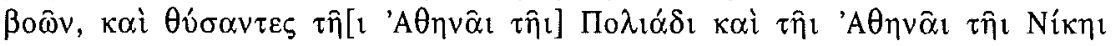

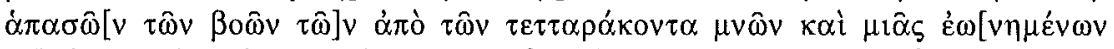

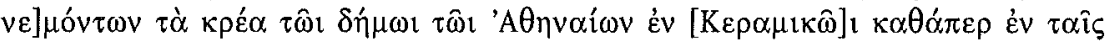

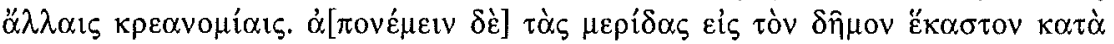

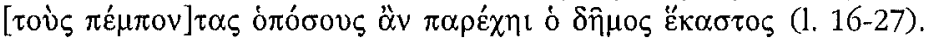

Que les hiéropes, après avoir acheté les vaches ${ }^{50}$ de concert avec les boônai ${ }^{51}$ avec les quarante et une mines qui proviennent de la location de Néa, une fois la procession faite en l'honneur de la déesse, immoleront toutes ces vaches auprès du grand autel d'Athéna, en réservant une des plus belles victimes pour Athéna Niké; qu'après avoir fait ce sacrifice à Athéna Polias et à Athéna Niké, ils distribueront au peuple athénien, au Céramique, les chairs de toutes les vaches achetées avec les quarante et une mines, comme cela se fait pour les autres distributions de chairs, en répartissant les portions entre les différents dèmes d'après le nombre de citoyens que chaque dème aura délégué à la procession ${ }^{52}$.

Il faut au peuple une raison pour légiférer en matière liturgique. Quelque chose a été modifié. Des changements peuvent intervenir dans les distributions de chair, mais elles sont mineures, pour le reste, on suivra les usages habituels en cette matière (même si l'on prend soin de préciser de quelle façon on procèdera entre les dèmes). Ce qui retient surtout l'attention des promoteurs du décret, c'est l'approvisionnement en animaux de sacrifice pour cette partie du programme sacrificiel des petites Panathénées, la partie certainement la plus coûteuse; la cité doit régler une question qui a dû faire problème Pour une raison qui nous échappe, on a dû manquer pendant un certain temps de tout ou partie des ressources dont on avait disposé (perte pour une raison quelconque des revenus d'un (ou plusieurs) temenos(e) , tarissement de fonds publics...) pour que soit dignement fêtée la déesse. En 1985, j'avais suivi l'explication qu'avait donnée L. Robert de l'origine des subsides que se propose d'utiliser le décret. Il voyait dans cette nea un nouveau territoire ${ }^{53}$. Il s'agissait, pensait-il, d'une partie du territoire d'Oropos qu'à cette date Philippe II avait offerte à Athènes. J'en doute un peu aujourd'hui, considérant, avec M.K.

50 Ce sont bien des vaches : tas bous; répété 1. 29.

51 Sur cette magistrature on verra la mise au point de V.J. Rosivach, Public Sacrifice, op. cit. (n. 10), p. $108 \mathrm{sq}$.

52 Traduction Durrbach, comme ci-dessus, à quelques mots près.

53 Voir n. 10. 
Langdon, qu'il est difficile d'imaginer les Athéniens appelant autrement que par son nom la terre qu'ils se voyaient attribuer. La localisation et la nature de Néa (plutôt un nom de lieu) restent une énigme, dont la solution, heureusement, n'engage pas la suite de notre étude. Ces 41 mines fournissent à la cité les moyens de financer le grand sacrifice à Athéna Polias sur le grand autel ${ }^{54}$.

La conception du programme sacrificiel que j'ai présentée plus haut, avec ses parts d'honneur du sacrifice de l'« ancien sanctuaire » et ses parts isonomes du grand autel ne repose pas seulement sur une appréciation qualitative des participants à chacun des partages, mais aussi sur les aspects quantitatifs de cette organisation. C'est à ces aspects quantitatifs que sont consacrées les remarques qui suivent.

Il serait évidemment très utile de savoir combien d'animaux pouvaient être achetés avec les revenus de Néa. Malheureusement, malgré des efforts récents $^{55}$, nos connaissances sur le prix des bovins restent notoirement insuffisantes; on ne dispose que de quelques prix, trop éloignés les uns des autres : 40 à 50 drachmes dans le calendrier de Thorikos vers $440^{56}$, en 414 , dans la vente des biens des Hermocopides, de 35 à 50 drachmes $^{57}$, environ $51 \mathrm{dr}$. en $410 / 9^{58}, 90 \mathrm{dr}$. dans le calendrier de la Tétrapole de Marathon au IVe siècle ${ }^{59}$. Mais, à partir des années soixante-dix, les mentions plus nombreuses donnent des valeurs plus stables: 70 drachmes dans le premier compromis des Salaminiens en $363 / 2^{60}$, dans les achats pour l'amphictyonie délienne ${ }^{61}$, à $\mathrm{Kym}^{62}$. Je propose de retenir le prix chronologiquement le plus proche de 70

54 La question qui agite beaucoup V.J. Rosivach : à savoir ce qui, dans l'inscription, correspond à ce qu'on appelle, dans d'autres sources, l'hécatombe, ne me semble pas fondamentale. Quoi qu'il en soit, je me trouve presque constamment en opposition avec ses analyses sur ce point, si bien que s'il fallait que je désigne un lieu pour l'hécatombe, ce serait, évidemment, le grand autel d'Athéna, alors qu'il la place dans, ou près de, l'« ancien temple » (art. cit. [n. 7], p. 440 sq.).

55 Tableaux de prix d'animaux sacrifiés dans deux excellentes études avec références : M.J. JAMESON, Sacrifice and Animal Husbandry in Classical Greece, in C.R. WITTAKER (éd.), Pastoral Economies in Classical Antiquity, Cambridge, 1988 (PCPbS, Suppl. 14,), p. 87-119, ici p. 91, et F. VAN STRATEN, Greek Sacrificial Representations: Livestock Prices and Religious Mentality, in Gifts to the Gods, Proceedings of the Uppsala Symposium 1985, 1987 (Acta Uniusitet Uppsala, Boreas 15), p. 159-170, ici p. 166 (plus complet); on doit voir aussi V.J. RosivaCH, Public Sacrifice, op. cit. (n. 10), p. 95 s. et L. ROBERT, Opera Minora, II, p. 780.

56 Datée de la première moitié du IVe s. par l'éditeur G. Daux, l'inscription a vu sa date très nettement remontée par D.M. Lewis : c. 440 (ZPE, 60 [1985], p. 108 n.3).

57 W.K. PRITChETT, The Attic Stelai Part II, in Hesperia, 25 (1956), p. 196-198, 257 (les circonstances ont pu faire que ces prix soient plus bas que la moyenne du moment).

$58 I G, \mathrm{I}^{3}, 375,1.7$ : on dépense cette année-là $5114 \mathrm{dr}$. pour le sacrifice des grandes Panathénées; le prix indiqué vaut pour le sacrifice de 100 bêtes, mais il pourrait varier beaucoup selon qu'elles pouvaient être plus ou moins nombreuses.

$59 I G, I^{2}, 1358 ; L S C G, 20$. Pas de possibilité de plus grand précision chronologique. Tous les bœufs y coûtent ce prix, à l'exception d'un (B. 1. 8) qui coûte $150 \mathrm{dr}$.

$60 \quad$ LSS, 19.

$61 I G, \mathrm{II}^{2}, 1635,1.35-36$.

62 I. Kyme, 13, 43-45. 
drachmes, valeur sur laquelle je fonde les calculs suivants ${ }^{63}$. À ce prix on a $\mathrm{pu}$ acheter environ 60 vaches. Si l'on estime le poids vif de tels animaux à $220 \mathrm{~kg}^{64}$, d'où un poids de viande net d'environ $110 \mathrm{~kg}$, on en tire que les Athéniens disposaient vers 330 de 6,5 à 7 tonnes de viande pour le grand partage isonomique panathénaïque annuel. C'est gigantesque, mais c'est à la mesure de la cité.

Nous connaissons le dividende (les 41 mines donnent $n$ bovins). Si le partage était conçu de telle façon qu'il permette à tout citoyen, dès lors qu'il participe, de disposer d'une part, la valeur de celle-ci peut être aisément calculée. Dans une telle configuration, en effet, il était plus prudent de prévoir le plus pour, éventuellement, satisfaire le moins. Il s'agirait donc de faire correspondre les ayants droit à l'ensemble de la population citoyenne adulte. Si nous nous plaçons donc dans cette hypothèse «maximaliste», et si nous nous servons de l'effectif de citoyens du recensement de Démétrios de Phalère, à une petite vingtaine d'années de là, à savoir 21000 citoyens adultes, nous obtenons une valeur pondérale de la part située entre 0,325 et $0,350 \mathrm{~kg}^{65}$, ou un peu plus si l'on tient compte de la viande mise de côté après le premier partage; disons donc $0,350 \mathrm{~kg}$. Il s'agit, en quelque sorte, d'une valeur plancher. Il est difficile d'aller plus loin et d'effectuer d'autres calculs, par exemple sur la population libre ou par oikos. Trop d'inconnues nous en empêchent ${ }^{66}$.

Mais il n'en allait probablement pas ainsi. D'abord parce qu'il aurait fallu que le diviseur fût connu, c'est-à-dire qu'au moins périodiquement on ait procédé au recensement du peuple athénien. On sait qu'il n'en est rien. Ensuite parce que, contrairement à ce qu'illustre cette façon de poser le problème, les interfaces ne sont pas constitués par la cité d'un côté et la foule des citoyens de l'autre, mais par la cité et ses subdivisions. Enfin, tout le monde n'accède pas au partage de la deuxième espèce, même si chacun est en puissance d'y parvenir. Il en va de même pour la procession.

Reprenons le texte. Il nous invite à inclure le grand sacrifice isonomique panathénaïque dans une série : katbaper en tais allais kreanomiais. Cela

63 À Délos, à la fin du IVe s. et à l'époque helténistique, on se tient près de ce montant : 70 drachmes. Voir sur ces questions le dossier mis en perspective par M.J. JAMESON, art. cit. (n. 55), p. 96 et l'appendice p. 107-111.

64 Je choisis un poids sur pied très légèrement supérieur à celui que proposent R.E. SLOAN et A.M. DUNCAN (Zooarcbaeology of Nicboria, in G. RAPP et S.E. ASCHENBRENNER [éds], Excavations at Nichoria in Southwest Greece, vol, 1, 1978, p. 60-77), et que reprend M.H. JAMESON (art. cit. [n. 55], p. 95), pour un site des Ages sombres, et cela, pour tenir compte des progrès accomplis durant près d'un demi-millénaire dans les domaines de la sélection et de l'alimentation.

65 Je divise le poids estimé plus haut non par 21000 , mais par 20000 pour tenir compte de l'effectif que j'estime à environ à quelques centaines de personnes qui participent au premier partage (cf. infra).

66 Je pense en particulier au célibat, aux différences de l'importance du régime carné selon l'âge, selon le sexe. 
signifie qu'il existe un ensemble de règles qui s'appliquent aux distributions de viande pour ce type de sacrifice politique; et l'on comprend bien qu'il ait été nécessaire d'en fixer. Dans la mesure où, d'une fête à l'autre, d'une certaine définition des bénéficiaires de la distribution à une autre, d'un dieu à l'autre, d'une catégorie de bétail à l'autre, la pratique sacrificielle pouvait être fort diversifiée, il convenait que les mageiroi puissent organiser le traitement des masses de viande de telle façon qu'elles fussent, si ce n'est mesurables, au moins comparables.

Le texte est clair sur ce point, la cité ne se préoccupe pas de répartir la viande entre les citoyens, mais entre les dèmes, et je suppose qu'il en allait de même dans ces autres distributions de chair auxquelles le texte fait référence avant. Et c'est là le point fondamental. Ce n'est pas en tant qu'individu que l'on participe à la procession puis au banquet sacrificiel qui clôt l'immolation des vaches du grand autel, c'est comme membre d'une collectivité politique, ce qui s'exprime par une organisation du partage qui s'appuie sur des cadres de nature politique. Si l'un d'entre eux apparaît ici clairement, le dème, je ne pense pas qu'il soit le seul, il y a tout lieu de croire que les dèmes sont rangés par tribus. Le processus mis en œuvre ne consiste donc pas en un traitement indifférencié d'une montagne de viande dans le but de la diviser d'emblée en un très grand nombre de parts, mais en opérations de divisions successives. On définit d'abord aisément la part de chaque tribu ${ }^{67}$ (ici , selon notre estimation, environ $660 \mathrm{~kg}$ de viande nette) puis, dans une opération plus complexe, et qui relève de chaque tribu considérée individuellement, il est distribué à chaque dème la quantité de viande qui lui revient, sous la responsabilité du démarque $^{68}$. Peut-être les trittyes entrent-elles alors en jeu. Peut-être le hiérope tribal joue-t-il alors un rôle.

Si nous prenons le cas moyen d'une tribu de 15 dèmes et que, par hypothèse, nous faisions comme si la représentation de chacun était identique, la moyenne de ce qui serait remis à chaque démarque s'établirait à $44 \mathrm{~kg}$ de viande. Mais ce n'est que pour donner une idée et pour rendre l'opération plus facile. Le nombre des démotes participant à la procession pouvait en réalité varier fortement d'un dème à l'autre, en particulier en raison de l'éloignement et de la densité de population. On devait donc avoir recours à une règle de trois pour parvenir au résultat recherché. Il fallait évaluer le nombre total de

67 On ne peut manquer de penser là à cette inscription d'Ilion, qui rend compte d'une fondation religieuse par un certain Hermias ( $I K$ Ilion, 52; principal commentaire par J. VANSEVEREN, in $R P b, 26$ [1936], p. 249-267), où les différentes pbylai se voient remettre une même somme d'argent pour l'achat d'un mouton qui sera sacrifié à Zeus Polieus et une vache pour Athéna; chacune des bêtes est identifiée par une marque permettant de reconnaître la tribu à laquelle elle correspond. Dans la procession, les phylarques doivent suivre les animaux qui leur ont été confiés.

68 Cf. la Souda au mot démarque, selon laquelle « ils organisent la procession des Panathénées "; $c f$. aussi la scholie à ARISTOPHANE, Nuées, 37, qui explique : "Clisthène ayant remplacé les naucrares par les démarques". Sur les rôles religieux des démarques, voir D. WHITEHEAD, The demes of Attica, 508/7 - 250 B.C., Princeton, 1986, p. 127-128. 
participant de la tribu, en se fondant, j'imagine, sur les déclarations des démarques; on divisait ensuite le lot qui était revenu à la tribu par cet effectif, ce qui donnait la valeur de la part (ou valeur de la part tribale puisqu'elle différait d'une tribu à l'autre) : $660 \mathrm{~kg} / \mathrm{x}$; il convenait pour finir de remettre au démarque $n$ fois cette valeur ( $n$ représentant le nombre de démotes ayant participé au cortège). Autrement dit, si $x$ est le nombre de participants à la pompè dans une tribu et $n$ le nombre de participants dans un dème de cette tribu, la quantité de viande remise à ce dème s'élève à $660 / x \times n$.

Si le scénario que je propose correspond à la réalité, le processus de partage comprendrait deux phases : la première, qui « découpe » la masse de viande disponible selon les jointures principales de la cité : ses divisions politiques, en l'occurrence les tribus, et qui aboutit à des quantités fixes (au moins aussi longtemps que le financement en bêtes de sacrifice n'est pas modifié) et égales; la seconde qui tient compte de la participation citoyenne et qui aboutit à fixer des parts, elles aussi égales, à l'intérieur de chaque tribu, mais de valeurs différentes d'une tribu à l'autre.

Au bout du compte, il est aujourd'hui impossible d'avancer une réponse à cette question simple mais difficile : de quelle quantité de viande disposait un citoyen qui participait à la procession et accédait, de cette façon, au banquet sacrificiel? Pour fixer, néanmoins les idées, rappelons que dans l'improbable hypothèse d'une participation de tous les Athéniens la part serait d'environ $0,350 \mathrm{~kg}$ et notons qu'avec un effectif de 1000 citoyens par tribu (soit, 70, en moyenne, par dème) on aboutit à des parts de $0,660 \mathrm{~kg} /$ personne.

Mais les groupes politiques considérés ont toute latitude pour organiser eux-mêmes leur participation ainsi que les opérations de redistribution. C'est ce que montre le décret du dème des Skambonidai (avant 460) où l'on voit les démotes légiférer sur leur participation aux Dipolies et aux Panathénées sur le partage des victimes ${ }^{69}$. Par ailleurs, quelques indices tendent à prouver que dans cette seconde partie de la procession des citoyens pouvaient se regrouper selon d'autres cercles de sociabilité. Comment comprendre autrement cette indication du calendrier du compromis entre les Salaminiens où un porc mâle de $40 \mathrm{dr}$. doit être sacrifié par le génos lors des Panathénées (LSS, 19, 1. 88)? Est-ce le cas des seuls Salaminiens $?^{70}$

Pouvons-nous maintenant proposer une estimation pondérale des parts obtenues dans le premier complexe sacrificiel? Essayons d'abord d'évaluer l'effectif des bénéficiaires. Une centaine de magistrats d'un côté et environ 200

$69 I G, I^{2}, 188,17$ (que le terme «Panathénées » de l'inscription renvoie plutôt aux grandes Panathénées ne change pas le fond de la question).

70 Ésope témoigne de ce qu'on sacrifiait des petits verrats aux Panathénées : à un acheteur qui demande si une truie est féconde, le vendeur répond : «Oui, extraordinairement : aux Mystères elle enfante des femelles et aux Panathénées des mâles ». Comme l'acheteur était surpris de ce qu'il entendait, le créancier ajouta : "Cesse de t'étonner car cette truie te donnerait aussi des chevreaux aux Dionysies » (Fables, 10). Cela ne signifie pas obligatoirement qu'Athéna Polias ait été la destinatrice de ces sacrifices de porcins. Doit-on penser à Kourotrophe? 
personnes de l'autre, qui représentent les «Athéniens ayant fait partie du cortège », soit environ $300^{71}$. En me plaçant dans la perspective qu'ouvre l'interprétation de E. Pfühl, qui voit dans les 4 boufs et 4 moutons de la frise nord les victimes du sacrifice de l'« ancien sanctuaire », on disposait de $520 \mathrm{~kg}$ de viande pour le partage ${ }^{72}$. Le reste est beaucoup plus douteux. Nous savons que les parts des magistrats, nouvellement définies, et celle des «Athéniens ayant fait partie du cortège » sont différentes, mais il nous est évidemment impossible de savoir dans quelle mesure elles l'étaient. Nous devons donc nous contenter d'une moyenne. Elle s'établit à $1,7 \mathrm{~kg} /$ personne, soit 5 fois plus que la moyenne calculée plus haut pour le sacrifice isonomique. Malgré l'incertitude générale où nous sommes, une telle différence est, à mon sens, significative. Il fait peu de doute que les deux séries de sacrifices et les deux partages qui en résultent sont entre eux dans un rapport tel que qualité et quantité y varient en sens inverse. Peu d'animaux, beaucoup de viande pour les bappy few, beaucoup d'animaux, moins de viande pour le nombre. Et, de nouveau, émerge cette image d'un seul corps animal résumant l'ensemble du processus, d'où l'on tirerait part divine, part d'honneur et part des hommes.

Tout ce détour, comme nous l'avions annoncé liminairement, pour revenir à la procession. De cette composition et de cet ordonnancement des sacrifices sur l'Acropole on passe sans difficulté à la composition et à l'ordonnancement de la procession. En tête : les magistrats, les canéphores et toutes les catégories de «porteurs » et de «porteuses», les transporteurs de biéra; puis les victimes des deux séries de sacrifices et les musiciens. En seconde partie, les Athéniens, répartis par dème et par tribu; chaque groupe de démotes étant contrôlé par son démarque, et l'ensemble tribal (?) sous l'autorité d'un hiérope (1. 33-34: « [que les hiéropes] mettent en mouvement la procession dès le lever du soleil, en infligeant l'amende légale ( $e k$ tôn nomôn) à ceux qui se déroberaient à leurs ordres »).

Ce que je retiens surtout d'une telle organisation c'est qu'on n'y aperçoit pas de participation individuelle, que celui qui processionne s'agrège au groupe de base dont il fait partie pour être intégré à la communauté de ceux qui accèdent au partage sacrificiel.

71 Effectifs assurés : 50 prytanes, 9 archontes, 10 trésoriers, 10 hiéropes, 10 stratèges, 10 taxiarques, soit 99 magistrats. Pour les autres, on peut proposer des effectifs plus ou moins sûrs : 100 canéphores, 10 thallophores (?), plus un nombre indéterminé, mais probablement peu important, de skaphéphores, d'hydriaphores et de musiciens. L'effectif ne dépasse donc probablement pas 300 (je ne compte par les ergastines [qui seraient une centaine] parce que je ne conçois leur présence que lors des grandes Panathénées, en raison de la remise du péplos).

$724 \times 110 \mathrm{~kg}$ ( $c f$. ci-dessus p. 54) de viande bovine: $440 \mathrm{~kg}$, auxquels s'ajoutent $4 \times 20 \mathrm{~kg}$ de viande ovine (cf. JAMESON, art. cit. [n. 55], p. 95) : $80 \mathrm{~kg}$. 


\section{Grandes Panathénées}

La première partie de la procession de la fête pentétérique est organisée de la même façon que celle des petites Panathénées. Son développement revêt évidemment plus de faste; de nouveaux groupes apparaissent, comme les ergastines qui ont œuvré au péplos; les groupes en -phoros y sont probablement plus étoffé, peut-être plus spécialisés. Mais, au total, plus de ressemblances que de différences. La grande singularité, c'est évidemment le péplos. Son offrande s'adresse au xoanon de la Polias, elle fait donc partie de la liturgie de l'«ancien sanctuaire » et prend donc place, aussi longtemps que cela est matériellement possible, dans la première partie de la procession. La lecture "chronologique » de la frise invite même à croire qu'une fois qu'il avait été détaché du mât, il était porté en tête de la procession et que la série des rites acropolitains commençait par la remise du péplos, d'abord à la prêtresse d'Athéna, puis au xoanon. C'est dans la seconde partie de la procession que les choses changent. Qui participe et dans quels cadres; qui offre?

Une série d'inscriptions du Ve siècle fournit la trame d'une histoire à éclipse de la participation d'unités politiques étrangères à la procession et au sacrifice.

\section{L'outremer}

1) Le premier texte est un décret athénien ${ }^{73}$ de $453 / 2(?)^{74}$ voté pour mettre à la raison les Érythréens. Le texte commence la liste de leurs obliga-

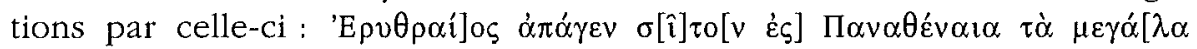

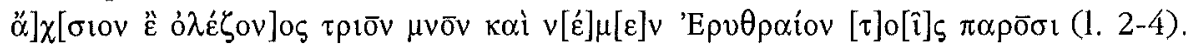
Malheureusement, la pierre et aussi la copie faite au début du XIXe siècle sont perdues, le seul fondement de nos réflexions est constitué par l'édition du CIG de Boeckh, et le doute subsiste; en particulier pour la question du grain. R. Meiggs et D.M. Lewis conviennent que le $\varsigma$ et l'o du siton sont signalés comme douteux par la copie, au témoignage de Boeckh; toutefois, ajoutent-ils, toutes les autres restitutions font encore plus violence à cette même copie (p. 91). En outre, les lignes suivantes semblent reprendre une obligation analogue faite aux Érythréens et on y lit : $\pi \rho i\langle\alpha\rangle \sigma \theta \alpha \imath$ $\hat{i}_{i}^{i}[\tau o v$. Prudents, Meiggs et Lewis concluent que les Érythréens se virent intimer l'obligation apporter du grain aux Grandes Panathénées ${ }^{75}$. J.-M. Bertrand comprend probablement le

$73 I G, \mathrm{I}^{3}, 14$ (R. MEIGGS - D. LEWIS, A Selection of Greek Historial Inscriptions, Oxford, 1980, $\mathrm{n}^{\circ}$ 40; M. Fornara, Translated Documents: Arcbaic Times to the Peloponnesian War; Baltimore, 1977, $\mathrm{n}^{\circ} 71$; J.-M. BERTRAND, Inscriptions bistorlques grecques, Paris, 1992, $\mathrm{n}^{\circ} 22$ ).

74 Controverse ancienne sur la date, renouvelée depuis la publication de nouveaux fragments de la liste du tribut de 454/3; pour une tapide prise de contact voir P. BRIANT, La guerre et la paix, in P. BRIANT - P. LÉVÊQUE, Le monde grec aux temps classiques. I : Le Ve siècle, Paris, 1994, p. 64.

75 Les auteurs des Atbenian Tribute Lists, Princeton, II, D 10 proposent des restitutions plus aventureuses. De là, la traduction de FORNARA (op. cit. [n. 731) : « The Sacrificial victims] sball be brought [by the Erythae]ans [in their entirety] to [the] Great Panathenaia (and they shall have the) 
texte de la même façon puisqu'il traduit : «Que les Érythréens fournissent du grain pour les Panathénées, trois mines au moins, et qu'il soit distribué aux Érythréens présents » (p. 57). Même si c'est la restitution la plus sage compte tenu de l'état du texte, il faut dire qu'historiquement cette obligation constitue pour nous un hapax dans la mesure où elle fait exception dans la série des obligations faites par les Athéniens aux membres de la Ligue de Délos. Dans un contexte très lacunaire, la mention des hiéropes un peu plus loin dans l'inscription invite à penser qu'à cette obligation concernant le grain s'en ajoutait d'autres en rapport avec les Panathénées.

2) Le texte suivant, dans sa datation la plus communément acceptée (mais non assurée !) est le décret de Kleinias ${ }^{76}$. La forme des lettres et l'identité de l'auteur de la proposition, s'il s'agit bien du père d'Alcibiade, poussent certains (Wade-Gery, Raubitschek) à remonter aux années quarante (447) un texte qui avait d'abord été placé au début des années vingt. Bien que méritant considération, ces arguments ne sont pas décisifs et les années trente pourraient bien

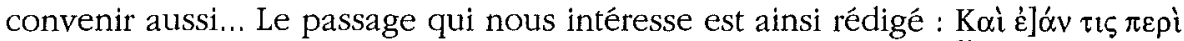

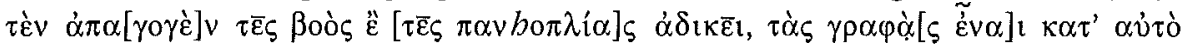

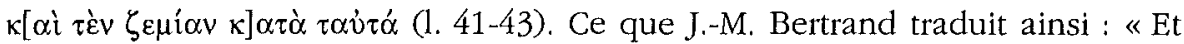
si quelqu'un commet une faute concernant le convoyage de la vache ou de la panoplie $^{77}$, que son procès soit mené de la même façon, et que la peine soit la même » (p. 59). Le texte fait ici référence à la procédure et à la peine ou l'amende à appliquer pour les fautes constatées dans la perception du tribut. Il est fort regrettable de ne pouvoir dater ce texte, même à quelques années près, avec quelque confiance : cela nous fournirait un terminus ante quem pour l'institution de l'obligation pour les cités alliées de la Ligue d'apporter un bœuf et une panoplie pour les Grandes Panathénées; ce décret présente en effet cette contrainte comme un usage déjà établi. L'usage de l'article, que l'on ne retrouve pas dans le décret de Thoudippos (infra), montre que tout le monde sait quelle est cette vache et quelle est cette panoplie.

3) Le décret de fondation de Bréa ${ }^{78}$ n'est pas non plus fermement fixé dans le temps. Je me range à l'opinion commune qui le place au milieu des années quarante. Au nombre des obligations qui sont faites aux colons qui vont partir, les Athéniens font figurer cette clause : qu'ils « envoient une vache et une panoplie ( $\alpha \alpha i \pi\left[\alpha v b o \pi \lambda i \alpha v^{79} \alpha \dot{\alpha} \alpha \dot{\alpha} \gamma \varepsilon v\right.$ ) aux Grandes Panathénées et un

value [of not less] than three minas, and distribution shall be made to those Erythraeans who are present».

$76 I G, \mathrm{I}^{3}, 34$ (MEIGGS-LEWIS, op. cit. [n. 73], $\mathrm{n}^{\circ}$ 46; BERTRAND, op. cit. [n. 73], $\mathrm{n}^{\circ} 23$ ).

77 Pour le supplément, voir le texte de Bréa ci-dessous.

$78 I G, \mathrm{I}^{3}, 46$ (MEIGGS-LEWIS, op. cit. [n. 73], n 49; BERTRAND, op. cit. [n. 73], $\mathrm{n}^{\circ} 24$ ).

79 On pourra s'étonner en ne considérant que les textes cités ici que cette restitution soit proposée sans commentaire. En effet, outre la vache, ce qui subsiste des documents disponibles pour évoquer les offrandes des colonies et des alliés laisse très largement place au doute : ici le $\pi$ initial, là un malheureux $\lambda$ au milieu d'importantes lacunes, ailleurs, enfin, rien! La solution à toutes ces énigmes a été trouvée par A. WiLheLM (GGA, 1903, 774) qui a rapproché de cet ensemble 
phallus aux Dionysies » (1. 11-13). Le fait nous était déjà connu : les colonies athéniennes participaient à la procession et au grand sacrifice panathénaïques, et cela, grâce au témoignage d'une scholie aux Nuées d'Aristophane (386) où l'on lit que toutes les «poleis" (!) (dèmes) de l'Attique ainsi que toutes celles qui ont été fondées par les Athéniens (apoikiai) doivent envoyer un bouf et processionner avec lui aux Panathénées. Le texte ajoute aussi des betera biéra qui sont peut-être, comme le veut L. Deubner (p. 34), des moutons pour Pandrosos.

4) Reste le dernier texte, postérieur, lui, à la réalisation de la frise, le décret de Thoudippos de $425 / 4$ portant modification de l'assiette du tribut ${ }^{80}$. Les passages qui nous intéressent concernent les impositions à venir. Premier décret, 1. 26 et suivantes : « les annonces concernant le tribut seront faites aux cités avant les grandes Panathénées » et, 1. 31-33: à l'avenir la nouvelle assiette sera établie tous les quatre ans pour les Panathénées; second décret, 1. 55-58: «Toutes les cités imposées durant l'année où Pleistias était le premier secrétaire de la boulè, sous l'archontat de Stratoklès, devront apporter un bœuf et une panoplie aux grandes Panathénées. Elles participeront à la procession [comme le font les apoikoi ?] ».

À la longue théorie des démotes, les grandes Panathénées ajoutent d'autres participants. Ce sont, d'abord, ces rameaux détachés du tronc de la métropole que sont les colonies et, probablement aussi, les clérouquies. Comment échapper alors à l'image des Panathénées comme fête «nationale » des Athéniens ? Mais il est clair que les Athéniens du Ve siècle, ceux qui régentent à leur guise la symmachie d'Athènes et ses alliés et tout ce qu'elle pouvait représenter de biens et d'intérêts communs, ont ajouté une obligation à toutes celles qu'ils faisaient par ailleurs à leurs alliés : l'ostensible démonstration de leur dévotion à l'Athéna Polias athénienne par leur insertion dans le rituel athénien commun. Tout se passe comme si les limites de l'Attique coïncidaient maintenant avec celle de l'Égée. Sont convoqués à la fête de la Vierge suprême tous ceux qui doivent lui savoir gré de sa protection. Et l'on pense, bien entendu, à ces traces diverses du prosélytisme «athénaïque » de la cité d'Athènes dans la seconde partie du siècle (en témoignent, pêle-mêle, les bornes de Samos, les monnaies de Cyzique et l'« Athéna-qui-protège-Athènes » d'Amorgos ${ }^{81}$.

Nouveauté éphémère d'un Ve siècle impérialiste? Pas totalement. L'histoire (résumée p. 44) des Épidauriens et de l'obligation de leur tribut annuel aux Athéniens lors des Panathénées (c'est mon interprétation), témoigne de ce qu'il ne s'agit pas d'une nouveauté, et que, dès le début du VIe

un document de Priène qui est une réponse à une «invitation » des Athéniens à se joindre aux

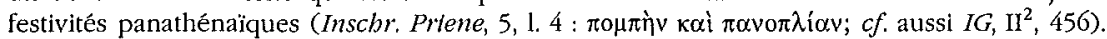

$80 I G, \mathrm{I}^{3}, 71$ (MEIGGS-LEWIS, op. cit. [n. 73], $\mathrm{n}^{\circ}$ 69).

81 Voir B. SMARCZYK, Untersucbung zur Religlonspolitik und politiscben Propaganda Athens im Deliscb-Attiscben Seebund, Münich, 1990. 
siècle ${ }^{82}$, les Athéniens pouvaient exiger qu'on s'acquitte d'une dette envers eux par une obligation faite aux débiteurs d'honorer leur divinité poliade. En d'autres temps, c'est en souvenir de l'ancienne syngenéia et de la pbilia que les Priéniens, après 326, acceptent l'invitation des Athéniens à venir chez eux processionner et apporter une panoplie aux grandes Panathénées ${ }^{83}$; les Colophoniens font de même en $307 / 6^{84}$.

À considérer ces documents dans leur ensemble, les choses sont claires, les alliés de la Ligue de Délos ont été tenus d'envoyer tous les quatre ans à Athènes une délégation de quelques personnes pour convoyer une vache, et d'apporter une panoplie à la grande fête de Pallas. Il leur est aussi demandé de participer à la procession. Il s'agit, bien entendu, de la seconde partie de celleci., qui comporte aussi, comme celle des Panathénées annuelles, une représentation du dèmos en ses composantes les plus fines : les dèmes. Un document de la fin du Ve siècle, montre peut-être qu'on a pu parfois rencontrer de sérieuses difficultés pour maintenir la continuité de l'offre des bovins par les dèmes durant la guerre du Péloponnèse. Les comptes des trésoriers d'Athéna enregistrent, pour l'année 410/9, le virement de $5115 \mathrm{dr}$. aux hiéropes annuels dans le but de financer l'achat d'animaux pour les grandes Panathénées ${ }^{85}$. On sait l'importance stratégique de la perte de Décélie en 413 et son occupation permanente par les Lacédémoniens. Pour en comprendre les conséquences, il n'est que de lire Thucydide : « Précédemment, les invasions, qui duraient peu... permettaient le reste du temps d'exploiter le sol; [à partir de l'occupation de Décélie], au contraire, avec l'ennemi installé à demeure...[celui-ci] mit le pays au pillage... Athènes se voyait privée de tout le pays... tous les troupeaux étaient perdus... En outre, le transport du ravitaillement venant d'Eubée qui, précédemment, au départ d'Oropos, s'opérait rapidement par terre en traversant Décélie, devenait par mer, en doublant Sounion, très onéreux » (Thuc., VII, 27, $3-28,1)$. Très onéreux, mais possible. Mais même cette voie fut coupée à partir de septembre 411, quand la flotte athénienne fut vaincue à Érétrie. La perte de l'accès à l'Eubée angoissait les Athéniens. Comment, dans de telle conditions, trouver les animaux nécessaires au sacrifice sans faire appel au budget de l'État et à des achats outre-mer ? Les comptes des trésoriers de $410 / 9$ prouvent, à mon sens, que la cité supplée les dèmes défaillants.

Des années cinquante aux années vingt du Ve siècle, Athènes peut assez régulièrement compter sur plus de 200 cités alliées et sur quelques dizaines de communautés issues d'elle-même (apoikiai et clérouquies); on ne risque donc pas d'exagérer le total en fixant à 220 l'effectif de ces délégations extérieures. S'y ajoutent les quelques 150 dèmes. Cela donne 370 vaches immolées sur le

\footnotetext{
82 Cf. mon étude citée n. 6, p. 24.

83 Cf. n. 79.

84 A. WILHELM, in JOAI Beib., 1 (1898), p. 45.

$85 I G, \mathrm{I}^{3}, 375$.
} 
grand autel! 40 tonnes de viande! Avant de telles quantités, des problèmes pratiques importants devaient trouver des solutions : l'hygiène, le charroi...

\section{La cité sous les armes}

Le contraste entre cette seconde partie et la première partie de la procession apparaîtra d'autant mieux quand on aura attiré l'attention sur un trait trop peu commenté : son caractère militaire. L'offrande de la panoplie : bouclier, casque, épée et lance peut être interprétée de plusieurs façons : comme rappel de la symmachie des communautés politiques déambulantes avec le peuple athénien, comme choix spécifique à destination de la déesse Stoichéia (IG, IV $\left.{ }^{2}, 487\right)$, Stratia (Plut., Préceptes politiques, 5e), Aréia, Sôtéria, Poliouchos ou Promachos, Promachorma ..., bref de la Vierge Lancière. Mais il y a plus.

Un des caractères particuliers des Panathénées, c'est que le peuple y processionne en armes. Les témoignages divers sur l'assassinat d'Hipparque par Harmodios et Aristogiton ne laissent pas de place au doute. On sait combien les versions d'Hérodote, de Thucydide et d'Aristote divergent, et sur de nombreux points. Selon Thucydide, si Harmodios, Aristogiton et les autres conjurés choisirent le cadre des Grandes Panathénées pour projeter d'assassiner Hippias, c'est parce que « c'était le seul jour où il fût possible aux citoyens qui devaient former le cortège de s'assembler en armes sans entraîner la méfiance » (VI, 56, 2). Il est question plus loin des « hoplites du cortège» $(58,1)$. Néanmoins, évoquant la façon dont les gardes d'Hippias furent chargés de mettre à l'écart ceux qui étaient soupçonnés de participer au complot, Thucydide évoque l'équipement normal du processionnaire, celui-ci ne comprenait pas l'ensemble des armes hoplitiques : « le bouclier et la lance étaient les seules armes qu'il était d'usage de porter dans les processions » $(58,2)$. Aristote conteste visiblement la version de Thucydide ou d'une autre source commune lorsqu'il écrit que « la tradition commune selon laquelle Hippias désarma les membres de la procession et découvrit ainsi ceux qui portaient des poignards est fausse; car alors on ne faisait pas la procession en armes; cet usage fut introduit plus tard par la démocratie » (A.P., 18, 4). L'opposition sur le déroulement des faits ne change rien à ce qui nous intéresse : au moins depuis la démocratie, c'est-àdire, du point de vue aristotélicien, au moins depuis Éphialte, mais probablement depuis bien plus longtemps si l'on fait, sur ce point, comme j'y suis enclin, plus confiance à Thucydide, le peuple athénien processionne en armes. Le peuple sous les armes, par dème et par tribu. Si l'on croit que les lexiarchika grammatéia tenus par les dèmes ont fourni la matière des rôles de mobilisation et que, dans la phalange, on combattait aux côtés de ceux qui avaient été inscrits la même année que soi sur le registre du dème, alors la montée vers la citadelle de Pallas emprunte sa structure, sa composition à l'armée du champ de bataille. C'est dans cette perspective qu'il convient, je crois, d'interpréter aussi la cavalerie de la frise. 
Tels sont les traits majeurs qui se dégagent des quelques textes importants de l'époque classique souvent négligés ou lus trop vite, et qui, on en conviendra aisément, ne se trouvent pas toujours en harmonie avec ce que montre la frise. Cette dernière pose plus de problèmes qu'elle n'en résout. Mais, comment s'en étonner si l'on garde à l'esprit que cette représentation devait se plier à la fois à des obligations esthétiques (ainsi la symétrie : comment représenter quelques animaux d'un côté et des dizaines de l'autre), à des règles narratives (comprimer le temps et l'espace pour tout dire en raccourci), et offrir, en même temps, des perspectives symboliques?

Pierre BRULÉ

Université de Rennes 2 Haute Bretagne

6 , avenue $G$. Berger

F - 35043 RENNES Cedex 